Chronic Obstructive Pulmonary Diseases: Journal of the COPD Foundation

\author{
Journal Club
}

\title{
The Journal Club: Computed Tomography and COPD
}

\author{
Ron Balkissoon, MD, MSc, DIH, FRCPC ${ }^{1}$
}

Abbreviations: chronic obstructive pulmonary disease, COPD; computed tomography, CT; forced expiratory volume in 1 second, FEV $\mathbf{1}$; Global initiative for chronic Obstructive Lung Disease, GOLD; Body mass index-airflow Obstruction-Dyspnea-Exercise capacity, BODE Citation: Balkissoon R. The journal club: Computed tomography and COPD. Chronic Obstr Pulm Dis. 2017;4(4):325-330. doi: https://doi. org/10.15326/jcopdf.4.4.2017.0174

1 Denver, Colorado

\section{Address correspondence to:}

Ron Balkissoon, MD, MSc, DIH, FRCPC

balkissoonr@njhealth.org

\section{Keywords:}

chronic obstructive pulmonary disease; COPD; computed tomography; CT; phenotypes; imaging; exacerbations; SPIROMICS

\section{Introduction}

COPD is recognized as a heterogeneous disease with contributions from airway inflammation and remodeling and parenchymal abnormalities. The small airways are perhaps the most vulnerable and earliest target for the injurious effects of cigarette smoke in susceptible individuals. Routine pulmonary function tests are limited in their capability of detecting early damage to the small airways and are limited in their ability to identify and or distinguish distinct COPD phenotypes. In this issue of Journal Club, we present 4 recent papers that provide examples of the ways in which we are expanding the use of computed tomography (CT) imaging to evaluate patients not only in terms of phenotyping but also follow up, prognosis and potentially early detection of susceptibility to developing more severe COPD. Ultimately, as we explore novel therapeutic interventions from pharmacotherapy, lung volume reduction surgery and even stem cell therapy, CT phenotyping may help to identify appropriate candidates. Indeed, one could argue it may ultimately be as instructive or more instructive than other biomarkers currently being studied. It is in this context that we now refer to CT biomarkers.

\section{Abstract 1 Recent Advances in CT Imaging in Chronic Obstructive Pulmonary Disease}

Bodduluri S, Reinhardt JM, Hoffman EA, Newell JD Jr, Bhatt SP. Ann Am Thorac Soc. 2017 [Epub ahead of print] Aug 16. doi: https://doi.org/10.1513/ AnnalsATS.201705-377FR

\begin{abstract}
Text:
Lung imaging is increasingly being used to diagnose, quantify and phenotype chronic obstructive pulmonary disease (COPD). Although spirometry is the gold standard for the diagnosis of COPD and for severity staging, the role of computed tomography (CT) imaging has expanded in both clinical practice and research. COPD is a heterogeneous disease with considerable variability in clinical features, radiographic disease, progression and outcomes. Recent studies have examined the utility of CT imaging in enhancing diagnostic certainty, improving phenotyping, predicting disease progression and prognostication, patient selection for intervention, and also in furthering our understanding of the complex pathophysiology of this disease. Multiple CT metrics show promise for use as imaging biomarkers in $\mathrm{COPD}$.
\end{abstract}

\section{Comments}

This is an excellent, state-of-the-art review of the literature regarding the multiple ways in which CT 
scanning is being explored to help us better understand and prognosticate when it comes to management of COPD patients. It is a general review of the advances made in $\mathrm{CT}$ imaging and their application to the diagnosis, evaluation, phenotyping and prognostic capabilities in COPD. The authors discuss the role of inspiratory and expiratory CT scans as a means of providing clinically relevant measures of COPD. It reviews $\mathrm{CT}$ densitometry as a way of assessing not only emphysema but also small airway disease. The authors also cover how CT scanning has allowed measurement of wall thickness for larger airways from the sixth generation upward. It outlines how regional ventilation studies allow the detection of significant regional variation in functional loss that is not detected by spirometry. It reviews the Jacobian determinant measurement that is an alternative $\mathrm{CT}$ registrationbased measure of regional ventilation. The authors also give an overview of the limitations of this technology as well as the practical considerations and discuss the clinical applications and future directions. For instance, they discuss how this technology will be very helpful with regards to selecting candidates for lung volume reduction surgery.

\section{Abstract 2 \\ Frequency of Exacerbations in Patients with Chronic Obstructive Pulmonary Disease: An Analysis of the SPIROMICS Cohort}

Han MK, Quibrera PM, Carretta EE, et al and

SPRIOMICS Investigators. Lancet Respir Med.

2017;5(8):619-626.

doi: https://doi.org/10.1016/S2213-2600(17)30207-2

\section{BACKGROUND:}

Present treatment strategies to stratify exacerbation risk in patients with chronic obstructive pulmonary disease (COPD) rely on a history of two or more events in the previous year. We aimed to understand year to year variability in exacerbations and factors associated with consistent exacerbations over time.

\section{METHODS:}

In this longitudinal, prospective analysis of exacerbations in the Subpopulations and Intermediate Outcome Measures in COPD Study (SPIROMICS) cohort, we analysed patients aged 40-80 years with COPD for whom 3 years of prospective data were available, identified through various means including care at academic and non-academic medical centres, word of mouth, and existing patient registries. Participants were enrolled in the study between Nov 12, 2010, and July 31, 2015. We classified patients according to yearly exacerbation frequency: no exacerbations in any year; one exacerbation in every year during 3 years of follow-up; and those with inconsistent exacerbations (individuals who had both years with exacerbations and years without during the 3 years of follow-up). Participants were characterised by the Global Initiative for Chronic Obstructive Lung Disease (GOLD) spirometric category (1-4) on the basis of post-bronchodilator FEV 1 . Stepwise logistic regression was used to compare factors associated with one or more acute exacerbations of COPD every year for 3 years versus no exacerbations in the same timeframe. Additionally, a stepwise zeroinflated negative binomial model was used to assess predictors of exacerbation count during follow-up in all patients with available data. Baseline symptom burden was assessed with the COPD assessment test. This trial is registered with ClinicalTrials.gov, number NCT01969344.

\section{FINDINGS:}

2981 patients were enrolled during the study. 1843 patients had COPD, of which 1105 patients had 3 years of complete, prospective follow-up data. 538 (49\%) of 1105 patients had at least one acute exacerbation during the 3 years of follow-up, whereas 567 (51\%) had none. 82 (7\%) of 1105 patients had at least one acute exacerbation each year, whereas only $23(2 \%)$ had two or more acute exacerbations in each year. An inconsistent pattern (both years with and without acute exacerbations) was common (456 [41\%] of the group), particularly among GOLD stages 3 and 4 patients (256 [56\%] of 456). In logistic regression, consistent acute exacerbations ( $\geq 1$ event per year for 3 years) were associated with higher baseline symptom burden, previous exacerbations, greater evidence of small airway abnormality on CT, lower interleukin-15 concentrations, and higher 
interleukin-8 concentrations, than were no acute exacerbations.

\section{INTERPRETATION:}

Although acute exacerbations are common, the exacerbation status of most individuals varies markedly from year to year. Among patients who had any acute exacerbation over 3 years, very few repeatedly had two or more events per year. In addition to symptoms and history of exacerbations in the year before study enrolment, we identified several novel biomarkers associated with consistent exacerbations, including CT-defined small airway abnormality, and interleukin-15 and interleukin-8 concentrations.

\section{FUNDING:}

National Institutes of Health, and National Heart, Lung, and Blood Institute.

\section{Comments}

The SPIROMICS investigators found that CT-defined small airway disease (assessed by the degree of air trapping) was associated with "consistent acute exacerbators" (defined as at least 1 acute exacerbation per year) during 3 years of prospective follow-up. The forced expiratory volume in 1 second ( $\mathrm{FEV}_{1}$ ) percentage predicted was associated with exacerbation rate in univariate analysis but it was not significant in multivariate analysis. The investigators primary target group for the study was the "frequent exacerbator" group ( $\geq 2$ exacerbations/year) but they actually found only about $2 \%$ of the individuals studied fit this criterion. As such, they were not able to find any correlations for this particular group. The significance of this finding is open to interpretation. The SPIROMICS cohort was recruited from academic centers and non-academic medical centers between 2010 and 2015. It would be interesting to know how many of the patients recruited for SPIROMICS were from the centers where these investigators are based. If so, this patient population may not be representative of the general COPD population in terms of their access to expert care. While they found no difference in exacerbation frequency between different types of centers over this period of time, many patients with severe to very severe COPD (Global initiative for chronic Obstructive Lung Disease [GOLD] stages III and $I^{1}$ ) would have presumably been on, or were put on, inhaled corticosteroids, long-acting beta2-agonists and/or long-acting muscarinic antagonists during the study. While it was a prospective observational study it appears that they did not control or track patient use of maintenance medications. The early GOLD guidelines recommendations are based on the presumption that patients are maintenance therapy naïve. This low prevalence number for frequent exacerbators may actually be a testament to the impact current standard therapies have in their ability to reduce exacerbations. While it is challenging to track the medications of this cohort over time it would have been interesting to see what maintenance therapies these patients were on over the 3- year period. It could further inform what should be the future therapeutic goals and outcomes to assess in patients on maintenance therapies.

\section{Abstract 3 Biomechanical CT Metrics Are Associated with Patient Outcomes in COPD}

Bodduluri S, Bhatt SP, Hoffman EA, et al and the COPDGene Investigators. Thorax. 2017; 72(5):409-414. doi: https://doi.org/10.1136/thoraxjnl-2016-209544

\section{BACKGROUND:}

Traditional metrics of lung disease such as those derived from spirometry and static single-volume $\mathrm{CT}$ images are used to explain respiratory morbidity in patients with COPD, but are insufficient. We hypothesised that the mean Jacobian determinant, a measure of local lung expansion and contraction with respiration, would contribute independently to clinically relevant functional outcomes.

\section{METHODS:}

We applied image registration techniques to paired inspiratory-expiratory CT scans and derived the Jacobian determinant of the deformation field between the two lung volumes to map local volume change with respiration. We analysed 490 participants with COPD with multivariable regression models to assess strengths of association between traditional CT metrics of disease and the Jacobian determinant with respiratory morbidity including dyspnoea (modified Medical Research Council), St Georges Respiratory Questionnaire (SGRQ) score, 6-min 
walk distance (6MWD) and the Body Mass Index, Airflow Obstruction, Dyspnoea and Exercise Capacity (BODE) index, as well as all-cause mortality.

\section{RESULTS:}

The Jacobian determinant was significantly associated with SGRQ (adjusted regression coefficient $\beta=-11.75,95 \%$ CI -21.6 to $-1.7 ; p=0.020$ ), and with 6MWD ( $\beta=321.15$, 95\% CI 134.1 to 508.1; $p<0.001$ ), independent of age, sex, race, body mass index, FEV1, smoking pack-years, CT emphysema, CT gas trapping, airway wall thickness and CT scanner type. The mean Jacobian determinant was also independently associated with the BODE index $(\beta=-0.41,95 \% \mathrm{CI}$ -0.80 to $-0.02 ; \mathrm{p}=0.039$ ) and mortality on follow-up (adjusted HR=4.26, 95\% CI 0.93 to 19.23; p=0.064).

\section{CONCLUSIONS:}

Biomechanical metrics representing local lung expansion and contraction improve prediction of respiratory morbidity and mortality and offer additional prognostic information beyond traditional measures of lung function and static single-volume CT metrics.

TRIAL REGISTRATION NUMBER:

NCT00608764; Post-results.

\section{Comments}

The Jacobian determinant analyzes inspiratory and expiratory scans at total lung capacity and functional residual capacity respectively and calculates the degree of lung deformation. The deformation map that is created by using this registration represents a point wise expansion and contraction of lung areas. It can detect regional ventilation and air trapping in a way that the FEV 1 cannot. They found that the Jacobian determinant was significantly associated with the St George's Respiratory Questionnaire and 6-minute walk distance as well as with the Body mass index-airflow Obstruction-Dyspnea-Exercise capacity (BODE) index and mortality. It is taking us to a new level in being able to assess these issues. The authors found that the effect size of the unit change in the Jacobian determinant is stronger than the effect sizes of static single volume CT measures of COPD such as emphysema percentage, gas trapping and airway wall thickness. The authors do point out that one of the limitations of their study was that the CT scans were not controlled for spirometry. Furthermore, they point out that it was a multicenter trial with several different scanners being used for image acquisition. This analysis promises greater ability to sub-phenotype COPD patients in ways that may help in terms of prognosis and potentially considering treatment options. While we all appreciate the limitations of the $\mathrm{FEV}_{1}$ in terms of being able to predict significant morbidity and/ or mortality, this new technology may allow more refined phenotyping and provide significant prognostic information beyond traditional measures of lung function and static single volume CT metrics with regard to respiratory morbidity and all-cause mortality. It would be interesting to see if it has improved sensitivity to detect responses to therapy. Although it may not be practical as a standard of clinical practice it would be interesting to incorporate in clinical studies to see if it is more sensitive than inspiratory capacity as compared to $\mathrm{FEV}_{1}$ patients.

\section{Abstract 4 \\ Total Airway Count on Computed Tomography and the Risk of COPD Progression: Findings from a Population-based Study}

Kirby M, Tanabe N,Tan WC, et al and the CanCOLD Collaborative Research Group and the Canadian Respiratory Research Network. Am J Respir Crit Care Med. 2017. [Epub ahead of print] Sep 8. doi: https://doi. org/10.1164/rccm.201704-0692OC.

\section{RATIONALE:}

Studies of excised lungs show that significant airway attrition in the 'quiet' zone occurs early in chronic obstructive pulmonary disease (COPD).

\section{OBJECTIVES:}

To determine if the total number of airways quantified in vivo using computed tomography (CT) reflects early airway-related disease changes and is associated with lung-function decline independent of emphysema in COPD.

\section{METHODS:}

Participants in the multi-centre, population-based, 
longitudinal CanCOLD study underwent inspiratory/ expiratory $\mathrm{CT}$ at visit-1; spirometry was performed at four visits over 6-years. Emphysema was quantified as the CT inspiratory-low-attenuation-areas-below -950HU (LAA950). CT total-airway-count (TAC) was measured as well as airway inner diameter and wall area using anatomically equivalent airways.

\section{MEASUREMENTS AND MAIN RESULTS:}

Participants included never-smokers ( $\mathrm{n}=286)$, smokers with normal spirometry at risk for COPD $(n=298)$, GOLD-I $(n=361)$ and GOLD-II COPD $(\mathrm{n}=239)$. TAC was significantly reduced by $19 \%$ in both GOLD-I and GOLD-II compared with neversmokers ( $p<0.0001)$ and by $17 \%$ in both GOLD-I and GOLD-II compared to at risk participants ( $p<0.0001)$ after adjusting for LAA950. Further analysis revealed parent airways with missing daughter branches had reduced inner diameters $(p<0.0001)$ and thinner walls $(p<0.0001)$ compared to those without missing daughter branches. Among all CT measures, TAC had the greatest influence on FEV1 $(p<0.0001), F_{1}$ / FVC $(p<0.0001)$ and bronchodilator responsiveness $(p<0.0001)$. TAC was independently associated with lung-function decline $\left(F E V_{1}: p=0.02 ; \quad F E V_{1} /\right.$ FVC:p=0.01).

\section{CONCLUSION:}

TAC may reflect the airway-related disease changes that accumulate in the 'quiet' zone in early/ mild COPD, indicating that TAC acquired with commerically-available software across various CT platforms may be a biomarker to predict accelerated COPD progression.

\section{KEYWORDS:}

computed tomography; copd; copd progression; emphysema; small airway disease PMID:28886252

\section{Comments}

From Dr. Jim Hogg's seminal work in evaluating surgical lung specimens from COPD patients, ${ }^{2}$ particularly from the National Emphysema Treatment Trial lung volume reduction study, ${ }^{3}$ we appreciate the focus of the small airways as a site of early, (often asymptomatic), injury for patients with COPD. This seems to appear before much of the emphysematous destruction is evident. The question for this group was whether or not the reduction in small airway number is reflected by changes in the more central airways. Their goal was to examine these issues in patients with early to mild COPD. The fact that these investigators could identify that there was a significant reduction in these airways in patients with mild COPD, independent of whether they demonstrate evidence of emphysema, suggests that this may be an early detection tool for individuals more susceptible to the effects of cigarette smoking. Of course, the major challenges will be in trying to balance the potential benefits of early detection and the cost of performing such $\mathrm{CT}$ scans in patients with a smoking history who may be relatively asymptomatic and show little decline in lung function. The question is: at what point does this become clinically relevant? One will need to weigh the cost versus the benefits of simply identifying these patients early for prevention strategies such as to get them to stop smoking. Furthermore, this may be one of the best ways to identify so called "rapid decliners" early on. This is a group where early intervention may have significant impact on changing the course of decline in lung function over time. To date it has been very challenging to identify this group even as newer medications become available that may be able to impact such things as airway remodeling. 


\section{References}

1. Global Initiative for Chronic Obstructive Lung Disease (GOLD). Global strategy for the diagnosis, management, and prevention of chronic obstructive pulmonary disease. GOLD website. Www. goldcopd.org. Published 2016. Accessed October 2017.

2. Hogg JC, Chu F, Utokaparch S, et al. The nature of small-airway obstruction in chronic obstructive pulmonary disease. New Eng J Med. 2004; 350:2645-2653.

doi: https://doi.org/10.1056/NEJMoa032158

3. National Emphysema Treatment Trial Research Group. A randomized trial comparing lung-volume-reduction surgery with medical therapy for severe emphysema. New Eng J Med. 2003; 348:2059-2073. 